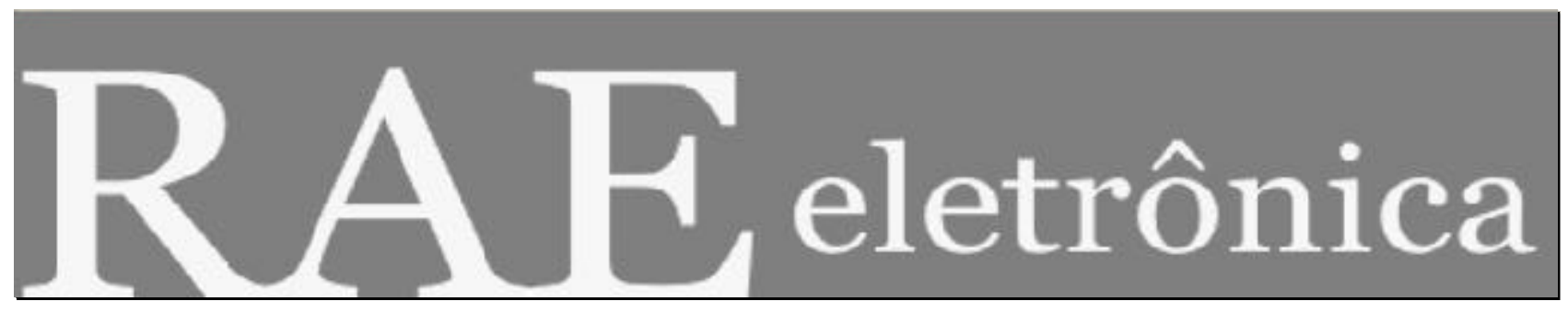

\title{
SELEÇÃO DE TÉCNICAS DE PREVISÃO DE MERCADO SEGUNDO AS DIFERENTES CATEGORIAS DE NOVOS PRODUTOS
}

Por:

Dario Amigo Sampron, UNITAU E UNIVAP (in memoriam)

RAE-eletrônica, v. 4, n. 2, Art. 22, jul./dez. 2005

http://www.rae.com.br/eletronica/index.c fm?FuseAction=Artigo $\& I D=2202 \&$ Secao=ARTIGOS $\&$ Volu $\mathrm{me}=4 \&$ Numero $=2 \& \mathrm{Ano}=2005$

CCopyright, 2005, RAE-eletrônica. Todos os direitos, inclusive de tradução, são reservados. É permitido citar parte de artigos sem autorização prévia desde que seja identificada a fonte. A reprodução total de artigos é proibida. Os artigos só devem ser usados para uso pessoal e nãocomercial. Em caso de dúvidas, consulte a redação: raeredacao@fgvsp.br.

A RAE-eletrônica é a revista on-line da FGV-EAESP, totalmente aberta e criada com o objetivo de agilizar a veiculação de trabalhos inéditos. Lançada em janeiro de 2002, com perfil acadêmico, é dedicada a professores, pesquisadores e estudantes. Para mais informações consulte o site www.rae.com.br/eletronica.

RAE-eletrônica ISSN 1676-5648

(C2005 Fundação Getulio Vargas - Escola de Administração de Empresas de São Paulo.

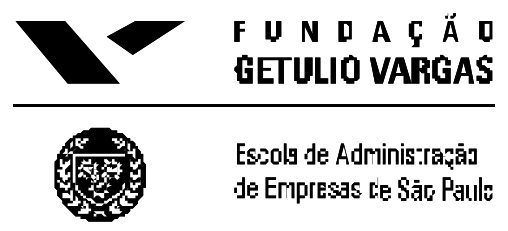




\title{
SELEÇÃO DE TÉCNICAS DE PREVISÃO DE MERCADO SEGUNDO AS DIFERENTES CATEGORIAS DE \\ NOVOS PRODUTOS \\ Dario Amigo Sampron
}

\section{RESUMO}

Apesar do grande número de técnicas disponíveis, o trabalho de previsão de mercado para novos produtos é difícil porque falta orientação adequada sobre quais técnicas utilizar. A maior parte da literatura sobre previsões de mercado para novos produtos enfatiza os aspectos matemáticos das ferramentas, e não as situações em que elas devem ser empregadas. Sendo assim, este artigo procura fornecer aos profissionais uma orientação sobre as técnicas mais aplicáveis a determinadas situações. A abordagem empregada considera a existência de diferentes categorias de novos produtos e as várias etapas que constituem um trabalho de previsão. Em razão de suas características e natureza, cada combinação de categoria de novo produto e etapa define conjuntos de técnicas de previsão aplicáveis. Também são apresentadas sugestões para futuras pesquisas.

\begin{abstract}
Market forecasting for new products is a difficult task. Some of the reasons are the large number of techniques available for forecasting and the scarce orientation on the best techniques to apply. Difficult increases because most of the literature on new product forecasting focuses on the techniques and not on the management issues, such as discussing when to employ the technique. This article aims to guide practitioners on the techniques to be applied in specific situations. The approach assumes there are different categories of new products and that a market forecasting is divided up in steps. Each combination of new product category and job step can define specific techniques to be adopted. Due to the extension of the subject, suggestions for future research are also indicated.
\end{abstract}

\section{PALAVRAS-CHAVE}

Técnicas de previsão, desenvolvimento de novos produtos, previsão de mercado, lançamento de produtos.

\section{KEYWORDS}

Forecasting techniques, new product development, market forecast, product introduction. 


\section{INTRODUÇÃO}

Novos produtos são essenciais para a manutenção da competitividade e para a obtenção de resultados positivos por parte das organizações. Essa importância é responsável por um grande número de lançamentos, exigindo dos administradores intenso envolvimento nas diversas fases do desenvolvimento de novos produtos.

Algumas das fases que integram o processo de desenvolvimento de um novo produto são fundamentais. Etzel, Walker e Stanton (2001) indicam que uma delas é a que determina a existência de um mercado suficiente para o produto, utilizada tanto para decidir sobre a viabilidade do projeto quanto para estabelecer prioridades entre vários produtos que estejam sendo cogitados.

Estimativas de mercado para novos produtos são obtid as por meio do emprego de técnicas de previsão e não deveriam constituir problema para os responsáveis pela previsão em razão da ampla variedade de técnicas disponíveis para realização do trabalho. No entanto, a escolha da técnica mais apropriada apresenta dificuldades. De acordo com Kahn (2002), isso ocorre porque a maior parte da literatura que trata das técnicas de previsão de mercado para novos produtos procura enfatizar aspectos matemáticos, não fornecendo indicação das situações em que elas sejam mais aplicáveis. Lynn, Schnaars e Skov (1999) também observaram essa particularidade, destacando que, apesar do grande número de técnicas disponíveis, não há uma clara orientação a respeito de quais sejam as mais efetivas para emprego em determinadas situações.

Lynn e Green (1998) destacaram também que, por serem poucos os produtos ou situações que permitem o emprego simultâneo de diversas técnicas, o benefício proporcionado pela existência dessa variedade é pequeno. Para novos produtos, especialmente os que não possuem similares no mercado, o trabalho de previsão se torna mais difícil quando comparado ao caso de produtos que, embora novos, possuem concorrentes que estejam sendo oferecidos no mercado. Isso se deve à inexistência de dados históricos, o que exclui a possibilidade de aplicação de muitas técnicas.

Tendo em vista a importância das previsões de mercado nas decisões relativas ao desenvolvimento de novos produtos, o presente artigo busca preencher a lacuna caracterizada pela carência de uma adequada orientação a respeito das situações de emprego de técnicas de previsão. Seu objetivo é auxiliar os administradores envolvidos em trabalhos de previsão de mercado para novos produtos na seleção das técnicas de previsão mais aplicáveis, tomando por base a situação tratada.

Este trabalho foi realizado essencialmente a partir de levantamento bibliográfico que permitiu destacar algumas das técnicas existentes e sua forma de classificação na tentativa de identificar situações de aplicação. O levantamento realizado serviu para a elaboração da proposta central deste artigo, que reconhece a influência de dois fatores na seleção de técnicas de previsão de mercado para novos produtos.

O primeiro fator é relativo à subdivisão de um novo produto em categorias. O segundo considera que um trabalho geral de previsão é realizado em etapas. Esses dois conceitos não são novos, embora sejam tratados isoladamente em partes diferentes da disciplina de marketing. Eles foram aqui 


\section{SELEÇÃO DE TÉCNICAS DE PREVISÃO DE MERCADO SEGUNDO AS DIFERENTES CATEGORIAS DE \\ NOVOS PRODUTOS \\ Dario Amigo Sampron}

combinados para caracterizarem situações condicionantes do emprego de técnicas de previsão. A abordagem empregada neste artigo procura destacar o vínculo e a importância desses dois fatores que, combinados, são determinantes para a seleção das técnicas de previsão de mercado de novos produtos.

\section{TÉCNICAS DE PREVISÃo DE MERCADO PARA NOVOS PRODUTOS}

As primeiras técnicas de previsão desenvolvidas envolviam procedimentos estatísticos. Francis Galton, na segunda metade do século XIX, apresentou um trabalho relacionando a altura de crianças às de seus parentes, o que se tornaria a base para o método de regressão (Galton, 1886). Posteriormente surgiram outras técnicas fundamentadas em estatística que permitem realizar previsões a partir de dados históricos. Entretanto, essas técnicas muitas vezes não podiam ser empregadas porque o caso não dispunha de séries históricas ou porque, quando estas estavam disponíveis, verificava-se que as condições passadas diferiam daquelas que vigorariam no período de previsão.

Nos anos de 1960 alguns pesquisadores, ao se defrontarem com essa limitação, passaram a desenvolver outras alternativas para a elaboração de previsões. O trabalho de Dalkey e Helmer (1963) é um exemplo, com relação ao método Delphi. O progresso em busca de ferramentas que dispensam o conhecimento histórico da variável a ser projetada pode ser verificado pelo trabalho de Reinmuth (1974), no qual é discutida a aplicabilidade de quatro técnicas que passaram a receber muita atenção por atenderem a esta necessidade: a obtenção de consenso em painéis de discussão, o Delphi, a previsão por analogia e as pesquisas de mercado. Nessa época, observou-se então um crescimento rápido do número de trabalhos sobre técnicas de previsão, conforme evidenciado pela análise de Armstrong (1986) correspondente ao período de 1960 a 1984.

Outra técnica importante desenvolvida nos anos de 1960 foi a de difusão. Ela considera que a disseminação de uma inovação em determinado mercado ocorre mediante um padrão, a saber: um novo produto é inicialmente aceito por um pequeno número de consumidores e, com o transcorrer do tempo, a demanda se amplia por efeito da comunicação de massa (influência externa) e pela divulgação boca a boca da inovação (influência interna) entre os consumidores. A demanda posteriormente se estabiliza e, por fim, decresce. Tal mod elo foi apresentado por Bass (1969) e utiliza equações diferenciais, sendo de particular utilidade na previsão de produtos inéditos.

Nos últimos 30 anos as pesquisas neste campo resultaram na disponibilidade de um número de técnicas relativamente elevado. Tal fato pode ser constatado pelo trabalho de Kahn (2002), que relaciona as 20 principais, acompanhadas de pequeno descritivo. Informações mais detalhadas sobre os conceitos e aspectos teóricos de cada técnica podem ser encontradas em livros-texto, como o de Makridakis, Wheelwright e Hyndman (1998).

Essa variedade de técnicas talvez tenha sido responsável pelo surgimento de uma forma de categorização com o propósito de fornecer uma orientação sobre as situações em que elas devem ser aplicadas. Consagrada entre muitos autores, essa categorização divide as técnicas em dois grupos. O primeiro é constituído por aquelas que se baseiam em avaliações ou opiniões pessoais para elaboração das previsões e, por essa razão, denominadas de "técnicas qualitativas" ou "por julgamento". O 


\section{SELEÇÃO DE TÉCNICAS DE PREVISÃO DE MERCADO SEGUNDO AS DIFERENTES CATEGORIAS DE \\ NOVOS PRODUTOS \\ Dario Amigo Sampron}

segundo, composto por aquelas que utilizam ferramentas matemáticas ou estatísticas, tem suas técnicas classificadas como "quantitativas".

Um comentário a respeito dessa classificação é que ela não parece ser absoluta, pois desperta dúvidas a respeito de como classificar determinada técnica. A opinião de especialistas, por exemplo, é reconhecida como uma técnica qualitativa. Entretanto, alguém também poderia considerá-la como quantitativa se os resultados estivessem amparados em extrapolações matemáticas. Igualmente, a pesquisa de intenção com consumidores pode ter dupla classificação, sendo considerada quantitativa ou qualitativa conforme as informações coletadas sejam numéricas ou não.

Embora amplamente reconhecida e adotada, essa forma de classificação mostra uma finalidade mais didática do que prática, pouco contribuindo para indicar as situações em que cada técnica seja mais aplicável. Em um primeiro momento, poder-se-ia concluir que as técnicas quantitativas, por empregarem dados históricos, seriam adequadas aos casos de mercados existentes, em que há produtos estabelecidos. Em contrapartida, as qualitativas seriam mais indicadas quando o ineditismo do produto fosse grande, situação esta caracterizada por não se dispor de dados passados. Essa separação também não é rigorosamente correta, pois as qualitativas podem ser empregadas tanto em um como em outro caso, posição esta registrada por alguns autores, tais como Sandhusen (1998).

\section{RECOMENDAÇÕES SOBRE A SELEÇÃO DE TÉCNICAS DE PREVISÃo}

Uma revisão da literatura revela tentativas no sentido de prover orientação sobre as técnicas mais adequadas para previsão de mercado de novos produtos. Um enfoque prático visando facilitar o trabalho dos profissionais envolvidos com previsões foi sugerido por Armstrong e Brodie (1999), que apresentaram um fluxograma no qual estão dispostos critérios a serem considerados na indicação da técnica mais aplicável.

De acordo com esse fluxograma, deve-se inicialmente avaliar se o caso em estudo apresenta disponibilidade de dados numéricos. Em caso positivo, recomenda-se o emprego de técnicas quantitativas. $\mathrm{O}$ passo seguinte consiste na verificação da existência de relações empíricas esperadas para o problema, como, por exemplo, as elasticidades-preço relativas ao produto. Assim, progressivamente, o responsável pela elaboração da previsão percorre os vários ramos do fluxograma, fornecendo respostas aos critérios estabelecidos em cada nó até obter uma indicação da técnica a ser empregada.

Esse procedimento, apesar de prático e simples, possui algumas limitações apontadas pelos próprios autores. Primeiramente há o reconhecimento de que ele não incorpora todos os critérios que permitam a obtenção de uma recomendação definitiva sobre a técnica a ser utilizada. Assim sendo, técnicas diferentes podem ser indicadas para um mesmo caso e, quando isso ocorrer, os autores sugerem que elas sejam utilizadas simultaneamente de modo a comparar os dois resultados. A segunda restrição se refere à situação em que o profissional responsável pelo trabalho de previsão não dispõe da resposta requerida em algum ponto do fluxograma e, desse modo, nenhuma indicação é apresentada. 


\section{SELEÇÃO DE TÉCNICAS DE PREVISÃO DE MERCADO SEGUNDO AS DIFERENTES CATEGORIAS DE NOVOS PRODUTOS \\ Dario Amigo Sampron}

Uma crítica adicional que pode ser feita a essa abordagem é que, apesar do esforço dos autores em estabelecer um procedimento abrangente, o fluxograma apresentado não incorpora determinados critérios relevantes no ambiente empresarial. Não há, por exemplo, nenhuma questão relativa à disponibilidade de recursos monetários e de tempo requeridos para a implementação de cada técnica. É de se esperar que essa ampla variedade de técnicas, pela diversidade apresentada, tenha custos e tempos de execução bem diferentes. Apesar das ressalvas apresentadas, esta abordagem possui grande valor por despertar para a necessidade de um procedimento que forneça indicações de quais técnicas de previsão são mais adequadas para a situação tratada.

Tais limitações podem ter sido responsáveis pela evolução de uma segunda forma de orientação, representada pelos trabalhos de Kahn (2002), de Lynn, Schnaars e Skov (1999) e de Kahn e Mentzer (1995). Esses autores realizaram pesquisas com executivos e profissionais envolvidos em previsões de mercado para novos produtos, procurando identificar quais técnicas são mais empregadas e relacioná-las a determinadas situações.

Algumas conclusões obtidas por Kahn (2002), extraídas de 168 respostas de uma pesquisa enviada a empresas norte-americanas de setores industriais dos ramos automotivo, médico, de saúde e de beleza, de equipamentos de construção e eletrônico, foram as seguintes:

- As técnicas mais empregadas são as de pesquisas de mercado com consumidores $(71 \%)$, opiniões de júri de executivos e de especialistas (53\%), composição da força de vendas (40\%) e analogia com outros produtos $(36 \%)$;

- Técnicas quantitativas são menos utilizadas do que as qualitativas, sendo as principais as linhas de tendência (19\%), média móvel (15\%) e amortecimento exponencial (10\%);

- Técnicas mais complexas do ponto de vista matemático, como Box-Jenkins e regressão nãolinear, foram pouco citadas, com $2 \%$ das preferências ou menos. Embora a pesquisa conduzida por Kahn (2002) tenha sido realizada de modo que os entrevistados apontassem as técnicas empregadas em função do tipo de novo produto, as respostas por tipo de novo produto não diferiram significativamente dos resultados gerais anteriormente detalhados. Isso mostra uma preferência generalizada, no âmbito empresarial daquele país, pelo uso de técnicas qualitativas em detrimento das quantitativas, mesmo quando o caso analisado dispunha de dados históricos, situação esta em que técnicas quantitativas são as mais indicadas.

Lynn, Schnaars e Skov (1999) utilizaram abordagem semelhante, investigando as preferências na utilização de determinadas técnicas de previsão, embora sua pesquisa tenha sido realizada em empresas do mercado industrial, cujos produtos apresentam diferentes níveis de conteúdo tecnológico. O trabalho desses autores, elaborado a partir da análise de 76 projetos de novos produtos, concluiu que empresas com produtos de alta tecnologia, cujos lançamentos haviam sido bem-sucedidos, empregavam com maior freqüência técnicas classificadas como qualitativas, com ênfase nas que aproveitam recursos internos da empresa para a obtenção das previsões.

Kahn e Mentzer (1995), seguindo a linha de pesquisa da opinião de profissionais de empresas, confirmaram que as práticas de previsão diferiam substancialmente das empresas industriais para as de consumo. Há preferência de empresas industriais pelo emprego da opinião de seus vendedores (composição da força de vendas) e que técnicas quantitativas (regressão linear, projeção de tendência e modelos econométricos) eram mais utilizadas em empresas voltadas para produtos de consumo. 


\section{SELEÇÃO DE TÉCNICAS DE PREVISÃO DE MERCADO SEGUNDO AS DIFERENTES CATEGORIAS DE \\ NOVOS PRODUTOS \\ Dario Amigo Sampron}

Outras conclusões obtidas a partir dos trabalhos anteriormente citados fornecem evidências adicionais. Armstrong e Brodie (1999) destacam existir a percepção entre os profissionais de que técnicas de maior complexidade matemática não fornecem resultados mais precisos. Kahn (2002) acrescenta que muitos responsáveis pela elaboração de previsões preferem utilizar técnicas de maior simplicidade e de mais fácil compreensão, com as quais os tomadores de decisão se sintam confortáveis, mesmo que a técnica escolhida não seja a mais efetiva para a situação tratada.

Kotler (2000) agrega aspectos importantes à discussão sobre a aplicabilidade de algumas técnicas ou grupos de técnicas, apontando limitações ou requisitos de algumas delas. Segundo o autor, pesquisas de intenção dos compradores, por envolverem trabalho de campo, demandam tempo e acarretam custos de implementação relativamente elevados, não sendo recomendáveis quando restrições de prazo e orçamento para realização do trabalho forem importantes.

Ainda de acordo com Kotler, a composição de opiniões da força de vendas será útil somente se a empresa tiver experiência no mercado e, em decorrência, possuir equipes de vendas estruturadas com informações e conhecimento relevantes desse mercado. Métodos quantitativos requerem dados de vendas passadas, os quais nem sempre estão disponíveis. Nesse caso, a opinião de especialistas é mais indicada, embora possa ser empregada também quando esses dados existirem, de modo a ter a possibilidade de confrontar seus resultados com os obtidos pela utilização de alguma técnica quantitativa.

Esse autor reconhece que a elaboração de previsões para um novo produto pode apresentar um menor grau de dificuldade quando concorrentes estiverem estabelecidos. A justificativa é que o trabalho será simplificado pela provável disponibilidade de dados históricos e pela existência de consumidores acostumados a avaliar e adquirir produtos semelhantes. Se a empresa tiver experiência no mercado a ser trabalhado, esse conhecimento deve ser aproveitado e poderá facilitar o trabalho de previsão. Em contrapartida, quando o mercado inexistir (produto com características altamente inovadoras), a previsão será dificultada pela carência de dados e pelo menor número de técnicas utilizáveis, embora o autor não explicite quais sejam elas.

Esse conjunto de proposições, obtido a partir da revisão da literatura, destaca alguns fatores importantes, tais como o tempo e o custo de utilização de cada técnica e o grau de ineditismo do novo produto como critérios para a seleção das técnicas de previsão aplicáveis.

\section{ETAPAS DE UM TRABALHO DE PR EVISÃO DE MERCADO}

Em geral, uma previsão de mercado não é obtida diretamente, em um único passo. A empresa normalmente possui competidores e a ela caberá uma fatia desse mercado, cuja dimensão dependerá de suas ações e da capacidade de reação de seus competidores. Mesmo quando a empresa é monopolista, é possível que a partir de uma data futura a presença de competidores oferecendo produtos similares tenha de ser considerada e, portanto, a parcela que lhe caberá desse mercado deverá ser prevista.

O trabalho de previsão compreende a realização de algumas etapas antes do objetivo final de determinação da participação do produto. Armstrong e Brodie (1999) sintetizaram quatro etapas 


\section{SELEÇÃO DE TÉCNICAS DE PREVISÃO DE MERCADO SEGUNDO AS DIFERENTES CATEGORIAS DE NOVOS PRODUTOS \\ Dario Amigo Sampron}

constituintes de um trabalho de previsão de mercado, indicando algumas técnicas que seriam mais aplicáveis em cada uma.

A primeira etapa de um trabalho de previsão compreende o dimensionamento do segmento para o qual o produto se destina. Pelo fato de o mercado ser influenciado por forças macroambientais, tais como condições econômicas e tendências sociais, as técnicas quantitativas são as mais indicadas, embora também possam ser utilizadas aquelas baseadas em julgamento (qualitativas). Entre as quantitativas, aqueles autores destacam a extrapolação de séries históricas de dados por terem menor custo. As técnicas econométricas são mais caras, mas, segundo os autores, costumam ser mais acuradas do que as que permitem fazer extrapolação e do que as que constituem o grupo "por julgamento". Desse grupo destacam o método Delphi como mais indicado para o caso de mercados novos ou para os existentes que apresentam rápidas alterações.

$\mathrm{Na}$ segunda etapa, procura-se prever as ações dos competidores estabelecidos ou de futuros competidores. Nela, de acordo com Armstrong e Brodie (1999), deve ser privilegiado o emprego de técnicas qualitativas, como a opinião de especialistas ou simulações que possibilitem concluir sobre as decisões tomadas pelos competidores em resposta às ações deflagradas pela empresa.

A terceira etapa objetiva a obtenção de uma previsão para a participação de mercado para o produto, partindo-se da avaliação da capacidade competitiva individual da empresa e da resposta de cada concorrente, prevista na etapa anterior. Nesse ponto eles recomendam o emprego de técnicas baseadas na opinião de especialistas, econométricas, de extrapolação de dados do mercado existente ou de previsão com base em mercados análogos ao que está sendo estudado.

Na última etapa, uma previsão de mercado para o produto é obtida multiplicando-se a participação de mercado determinada na etapa anterior pelo total do mercado, obtido na primeira etapa. Nela não são aplicáveis técnicas de previsão, uma vez que o resultado dessa etapa é obtido da interação dos resultados de duas etapas anteriores.

Ressalte-se que Armstrong e Brodie (1999) não exploraram o ineditismo do produto como elemento diferenciador ao discutirem cada etapa. Dessa forma não consideram que, em cada uma delas, as técnicas aplicáveis podem ser diferentes dependendo do grau de ineditismo do produto que está sendo tratado. Apenas reforçam que o trabalho de previsão para novos produtos é particularmente importante tendo em vista os substanciais investimentos demandados e os grandes prejuízos incorridos no caso de erros. Enfatizam também que o emprego de métodos que se baseiam em pesquisas de intenção de compra não é aplicável nesses casos pela falta de familiaridade dos consumidores com o produto e a conseqüente impossibilidade de se obterem opiniões confiáveis desse modo.

A abordagem utilizada por Armstrong e Brodie (1999), no entanto, destaca outro importante fator que deve ser considerado na seleção de técnicas de previsão. Trata-se da indicação de que o trabalho de previsão é constituído por etapas nas quais podem ser empregadas diferentes técnicas.

\section{IMPORTÂNCIA DAS CATEGORIAS DE NOVOS PRODUTOS NA SELEÇÃO DE TÉCNICAS DE PREVISÃO}




\section{SELEÇÃO DE TÉCNICAS DE PREVISÃO DE MERCADO SEGUNDO AS DIFERENTES CATEGORIAS DE \\ NOVOS PRODUTOS \\ Dario Amigo Sampron}

Diversos autores reconhecem o fato de não existir um único tipo de novo produto. De fato, novos produtos se diferenciam substancialmente, podendo ser classificados em categorias, a partir de determinados critérios. Mattar e Santos (2003), por exemplo, apresentam cinco categorias de novos produtos, diferenciando-os pelo grau de ineditismo que representam para as empresas que os desenvolvem e para o mercado aos quais se destinam.

Na primeira categoria se situam os lançamentos que correspondem a melhorias ou revisões de produtos já existentes. Nela estão incluídos também os que incorporam apenas reduções de custos, melhoria do desempenho ou alterações que elevem o valor destes para os consumidores. A segunda compreende os reposicionamentos no mercado. São produtos existentes que passam a ser oferecidos para outros mercados ou segmentos de mercados. A terceira categoria é a dos produtos que são novos para a empresa, mas que possuem similares no mercado em que a empresa pretende atuar. Na quarta consideram-se os produtos que são novos para a empresa, sendo igualmente novos no mercado em que a empresa pretende atuar, mas que possuem similares oferecidos em outros mercados. Finalmente, a quinta categoria abrange os produtos totalmente novos. São os casos que partem de idéias originais ou radicalmente inovadoras transformadas em produtos pela primeira vez.

Do ponto de vista de previsão, um extremo apresenta maior simplicidade, uma vez que a empresa possui ampla experiência do mercado e este é constituído por consumidores acostumados a adquirir produtos da empresa, antecessores deste, ou outros produtos concorrentes. Tal situação apresenta, em geral, para efeito de previsão, disponibilidade de dados históricos, conhecimento das sazonalidades e de outros fatores importantes a serem considerados na previsão do novo produto, o que pode ampliar o número de técnicas aplicáveis.

Em outro extremo está o caso mais complexo, pois o produto é totalmente novo e desconhecido tanto pela empresa quanto pelos consumidores. Analogia com produtos similares e experiência de concorrentes não podem ser aproveitadas na elaboração das previsões. Esses aspectos antecipam uma grande dificuldade para o trabalho e restrição quanto ao número de técnicas potencialmente aplicáveis. $\mathrm{O}$ fato de diferentes etapas de um trabalho de previsão exigir técnicas de previsão diferentes foi apontado anteriormente. Entretanto, não foram identificados estudos na literatura que apresentem indicações ou sirvam como guia para a seleção de técnicas de previsão que reconheçam a etapa de trabalho de previsão com a categoria de novo produto como condicionantes da escolha das técnicas.

Este trabalho sugere essa nova abordagem na qual, para efeito de seleção de técnicas de previsão de mercado de novos produtos, diferentes técnicas são aplicáveis conforme o grau de ineditismo do produto. Cada categoria de novo produto será confrontada com as etapas previstas para um trabalho de previsão definindo situações com particularidades capazes de influenciar a seleção de técnicas de previsão.

Cada situação é apresentada a seguir e foram definidas a partir de quatro categorias de novos produtos consideradas de importância para a seleção de técnicas de previsão. Para cada uma delas as várias etapas do trabalho de previsão são analisadas.

\section{Novo produto envolvendo simples melhorias ou reposicionamento no mercado}




\section{SELEÇÃO DE TÉCNICAS DE PREVISÃO DE MERCADO SEGUNDO AS DIFERENTES CATEGORIAS DE \\ NOVOS PRODUTOS \\ Dario Amigo Sampron}

De acordo com Mattar e Santos (2003), simples melhorias de produtos e reposicionamentos compreendem duas categorias distintas. Para efeito de emprego de técnicas de previsão, considerou-se que elas constituem uma mesma situação, recebendo então a mesma análise. Ambas se caracterizam primordialmente pela existência de um mercado em que a empresa já atue, o que implica haver consumidores familiarizados com o tipo de produto. A presença de concorrentes pode ocorrer ou não.

Com respeito à quantificação do mercado geral ou do segmento, objeto da primeira etapa do trabalho de previsão, todas as técnicas podem potencialmente ser empregadas, incluindo as de menor complexidade matemática, como as de regressão linear ou linha de tendência. Estas têm a vantagem de não requererem procedimentos ou softwares especiais, podendo ser operacionalizadas com planilhas de cálculo e baixo custo de implementação. A compreensão mais fácil, a melhor aceitação por parte dos tomadores de decisão e uma maior facilidade de encontrar profissionais capacitados a trabalhar com essas técnicas de menor complexidade são fatores favoráveis à sua adoção. Técnicas mais sofisticadas - por requerem a busca de profissionais ou de consultorias em que, em geral, se encontra maior especialização - podem onerar e retardar o trabalho de previsão.

A modificação do produto por parte da empresa corresponde ao caso ideal para realização de pesquisas entre os consumidores, as quais serão contra-indicadas se houver restrições de orçamento ou se, ainda, por razões estratégicas, a empresa visar maior sigilo de seu projeto. Nessa situação deve ser ponderado o risco de se realizar o desenvolvimento do produto sem consulta aos consumidores, mesmo que as modificações em relação ao produto existente sejam pequenas e que a empresa julgue ter ampla compreensão do mercado.

Se a empresa não for monopolista, que é o caso mais comum no ambiente atual, deve-se realizar a segunda etapa, na qual são previstas as reações dos concorrentes. Da mesma forma, uma previsão para a participação de mercado da empresa será necessária, sendo ela objeto da terceira etapa. Nessas duas etapas podem ser aplicadas, por exemplo, opiniões de especialistas ou Delphi. Para a previsão da participação, as técnicas que se baseiam em métodos econométricos e as de extrapolação também podem ser empregadas. Ressalve-se que, para o caso de mercados em que os participantes tenham atingido um nível de estabilidade de poder competitivo e para o qual não se vislumbre a entrada e a saída de competidores, as participações de mercado tendem a se manter próximas daquelas observadas no passado recente. Portanto, essa parte do trabalho de previsão não deverá apresentar maior dificuldade, podendo a empresa, aqui, tirar proveito das opiniões da força de vendas, processo relativamente simples, rápido e barato, por estar disponível internamente.

Se a empresa for monopolista e, pla existência de qualquer tipo de barreira, verificar-se a impossibilidade da entrada de novos concorrentes no setor, essas etapas do trabalho serão dispensadas. Nessa situação, o volume determinado para o mercado ou o segmento, conforme a primeira etapa, será o resultado da previsão para o produto em estudo.

\section{Novo produto para a empresa, destinado a mercado em que existem produtos similares}

Para o dimensionamento do mercado geral ou do segmento são válidas as recomendações feitas para o caso anterior, se dados históricos estiverem disponíveis. Do contrário, o profissional responsável pela previsão poderá empregar técnicas que utilizem a opinião de especialistas externos, uma vez que na empresa é provável não existir pessoal com conhecimento suficiente desse mercado. Como essa 


\section{SELEÇÃO DE TÉCNICAS DE PREVISÃO DE MERCADO SEGUNDO AS DIFERENTES CATEGORIAS DE NOVOS PRODUTOS \\ Dario Amigo Sampron}

situação compreende um mercado existente, em que produtos similares estão disponíveis, as pesquisas com os consumidores são aplicáveis.

Reações dos concorrentes devem ser previstas em resposta à ação da empresa de entrar nesse mercado. Para a elaboração dessa etapa do trabalho, a opinião de especialistas externos é altamente recomendável, uma vez que a existência do mercado significa a disponibilidade de profissionais com conhecimento adequado deste.

Com respeito à previsão de participação de mercado, se a empresa tiver um objetivo agressivo para ele, a distribuição das participações de alguns concorrentes deverá mudar significativamente em relação ao passado. A empresa, ao sair do zero e capturar uma fatia substancial do mercado, à custa dos demais concorrentes, provocará uma alteração geral na distribuição das participações, inviabilizando a obtenção de previsão dos valores individuais a partir de simples extrapolações de dados passados. Portanto, essa é uma situação típica para a utilização de opiniões externas, em particular se empregando o método Delphi porque ela procura convergir opiniões que, dado o grau de incerteza, tendem naturalmente a ser discordantes.

Se a empresa não tiver como meta uma grande participação no horizonte de previsão, de modo que suas ações não alterem substancialmente as participações dos demais concorrentes estabelecidos, a opinião de executivos da empresa ou da força de vendas poderia ser utilizada com vantagens por apresentar maior rapidez e menor custo. Entretanto, essa situação implica o desconhecimento do mercado pela empresa e, portanto, a utilização de recursos internos pode não ser confiável.

\section{Novo produto para a empresa e para o mercado, mas produtos similares de outras empresas são oferecidos em outros mercados}

Trata-se de um desafio maior para obtenção de previsões em comparação aos casos anteriormente analisados, pois o produto é novo para a empresa e ela, bem como seus concorrentes, desconhecem o mercado. Como não há dados históricos disponíveis para o mercado, a ênfase deve recair sobre as técnicas qualitativas, principalmente Delphi, em que se busca uma convergência de conclusões entre especialistas.

Um outro aspecto importante a considerar é a percepção de que a experiência existente de comercialização de produtos similares em outros mercados poderá ser aproveitada. Particularmente nesse caso também podem ser empregadas as técnicas de previsão por analogia e de difusão, pois existe a experiência do desempenho de produtos semelhantes, embora em outros mercados.

Para a análise das reações dos concorrentes, a opinião de especialistas externos também poderá ser de utilidade ao se elaborar a segunda etapa do trabalho de previsão. Em decorrência dessa análise, a empresa poderá ser considerada monopolista ou não, dependendo das barreiras à entrada de competidores no horizonte de previsão.

Se existirem concorrentes, previsões de participação tanto para a empresa quanto para os concorrentes considerados deverão ser obtidas na terceira etapa, com todos os concorrentes tendo uma participação inicial nula. As técnicas aqui aplicáveis seriam qualitativas, indicando-se Delphi, análises de cenários, simulações e pesquisas de mercado. Nesse caso a escolha final deverá obedecer a 


\section{SELEÇÃO DE TÉCNICAS DE PREVISÃO DE MERCADO SEGUNDO AS DIFERENTES CATEGORIAS DE \\ NOVOS PRODUTOS \\ Dario Amigo Sampron}

restrições como custo de utilização, tempo para obtenção de resultados, domínio da técnica e aceitação desta por parte dos tomadores de decisão.

\section{Novo produto para a empresa e para o mercado em geral}

Essa é a situação de maior grau de dificuldade para um trabalho de previsão, pois o produto é desconhecido pelos consumidores, que não estão familiarizados com os benefícios e as funcionalidades de produtos similares. O comportamento do mercado é uma incógnita para a empresa, que não pode utilizar como referência produtos de outros fabricantes ou a resposta observada em outros segmentos. Nessa situação o número de técnicas potencialmente utilizáveis para o trabalho de previsão de mercado é pequeno. A ausência de dados históricos de vendas de concorrentes e de produtos análogos sugere para o dimensionamento do segmento, objeto da primeira etapa, o emprego de técnicas qualitativas, sendo os modelos de difusão e o Delphi os mais indicados. Pesquisas de mercado apresentam desvantagens em virtude do desconhecimento do consumidor em termos do produto e de outros similares, o que não permite que a empresa obtenha resultados confiáveis. Opinião de executivos da empresa e composição da força de vendas - por explorarem o conhecimento interno, que no caso deve ser precário - são possibilidades que devem ser evitadas.

Ao menos que o produto seja de pequeno conteúdo tecnológico, facilmente imitável e com fracas barreiras à entrada de concorrentes, a participação de mercado prevista para o produto será total durante um largo horizonte e as etapas do trabalho de previsão em que se estimam as participações de eventuais concorrentes é desnecessária, reduzindo o trabalho de previsão ao dimensionamento do mercado geral. O Quadro 1 a seguir apresenta um resumo das recomendações apresentadas.

Quadro 1 - Resumo das técnicas de previsão indicadas de acordo com a categoria de novo produto e a etapa do trabalho de previsão

\begin{tabular}{|l|l|l|l|}
\hline Categoria/Etapa & Dimensionamento do segmento & $\begin{array}{l}\text { Previsão de ações dos } \\
\text { competidores }\end{array}$ & $\begin{array}{l}\text { Previsão de participação de } \\
\text { mercado para o produto }\end{array}$ \\
\hline $\begin{array}{l}\text { Novo produto envolvendo } \\
\text { simples melhorias ou } \\
\text { reposicionamento no mercado }\end{array}$ & $\begin{array}{l}\text { Todas, com preferência para as } \\
\text { mais simples em termos de } \\
\text { operacionalização e menor custo }\end{array}$ & $\begin{array}{l}\text { "Por julgamento", com } \\
\text { destaque para opiniões de } \\
\text { especialistas ou Delphi }\end{array}$ & $\begin{array}{l}\text { "Por julgamento", com } \\
\text { destaque para as opiniões da } \\
\text { força de vendas ou as técnicas } \\
\text { quantitativas baseadas em } \\
\text { métodos econométricos e de } \\
\text { extrapolação }\end{array}$ \\
\hline $\begin{array}{l}\text { Novo produto para a empresa, } \\
\text { destinado a mercado no qual } \\
\text { existem produtos similares }\end{array}$ & $\begin{array}{l}\text { Se houver dados históricos: } \\
\text { potencialmente todas; } \\
\text { se não houver dados: opinião de } \\
\text { especialistas externos e } \\
\text { pesquisas de mercado }\end{array}$ & $\begin{array}{l}\text { Opinião de especialistas } \\
\text { externos }\end{array}$ & $\begin{array}{l}\text { Opinião de especialistas } \\
\text { externos e Delphi }\end{array}$ \\
\hline
\end{tabular}




\begin{tabular}{|l|l|l|l|}
\hline $\begin{array}{l}\text { Novo produto para a empresa } \\
\text { e para o mercado, mas } \\
\text { produtos similares de outras } \\
\text { empresas são oferecidos em } \\
\text { outros mercados }\end{array}$ & $\begin{array}{l}\text { Delphi, analogia ou difusão } \\
\text { externos }\end{array}$ & $\begin{array}{l}\text { Técnicas qualitativas com } \\
\text { destaque para Delphi, análise de } \\
\text { cenários, simulações e } \\
\text { pesquisas de mercado }\end{array}$ \\
\hline $\begin{array}{l}\text { Novo produto para a empresa } \\
\text { e para o mercado em geral }\end{array}$ & $\begin{array}{l}\text { Técnicas qualitativas com } \\
\text { destaque para Delphi e modelos } \\
\text { de difusão }\end{array}$ & -- & - \\
\hline
\end{tabular}

\section{CONSIDERAÇÕES FINAIS}

Considerando a importância do trabalho de previsão de mercado de novos produtos, o grande número de técnicas disponíveis para previsões e a carência de indicações sobre a seleção das técnicas mais aplicáveis, é necessário o desenvolvimento de orientações para que os administradores possam realizar mais facilmente essa atividade.

A análise da literatura disponível mostra que determinadas técnicas são mais empregadas do que outras no ambiente empresarial, e os critérios de escolha são, geralmente, a praticidade oferecida aos responsáveis pela elaboração do trabalho de previsão e sua aceitabilidade por parte dos tomadores de decisão. Essa preferência recai sobre as técnicas tradicionalmente classificadas como qualitativas, ainda que os casos tratados disponham de dados em que as quantitativas poderiam ser empregadas.

Infelizmente os diversos artigos que apresentam levantamentos de opinião com respeito às técnicas mais empregadas não se referem ao ambiente empresarial brasileiro. A primeira recomendação que resulta deste estudo é que devem ser conduzidas pesquisas com respeito à situação nacional tendo em vista dois objetivos. O primeiro objetivo seria possibilitar uma comparação do estado de nossa realidade com a dos Estados Unidos, onde tais pesquisas normalmente são realizadas. Isso permitiria conhecer os critérios que as empresas em nosso país levam em consideração para selecionar técnicas de previsão de mercado ou como os especialistas consideram as diferentes categorias de novos produtos e as etapas do trabalho de previsão na seleção de técnicas. O segundo objetivo seria o de apoiar ou refutar as relações apresentadas no presente artigo, uma vez que ele não está embasado em evidências empíricas.

Uma segunda proposta desse artigo é a sugestão de aprimoramento de um fluxograma que oriente os especialistas na decisão sobre quais técnicas empregar em função da situação tratada. O fluxograma mencionado na revisão de literatura não demonstra estar em estágio suficientemente desenvolvido para ser empregado de modo seguro na indicação de técnicas de previsão. Basicamente isso se deve ao grande número de fatores que podem influenciar a escolha e o fato de que alguns não estão ali contemplados, como o prazo para a obtenção da previsão, os custos de implementação, a facilidade quanto ao seu domínio e emprego da técnica e a confiança por parte dos tomadores de decisão. Estes fatores são de importância no ambiente empresarial e também sua real influência precisaria ser mais bem investigada. 


\section{SELEÇÃO DE TÉCNICAS DE PREVISÃO DE MERCADO SEGUNDO AS DIFERENTES CATEGORIAS DE \\ NOVOS PRODUTOS \\ Dario Amigo Sampron}

É de se destacar que a literatura não abordou até o momento a seleção de técnicas de previsão conforme o grau de ineditismo do produto, não estando reconhecida uma relação entre categoria de novo produto e técnica mais aplicável. Espera-se que, com este artigo, tenha havido uma contribuição para o reconhecimento do ineditismo do produto como fator determinante na decisão da técnica a ser aplicada.

Previsões de mercado são importantes nas decisões de lançamento e para o desenvolvimento de um novo produto em todas as empresas. Particularmente para as que lidam com produtos industriais de grande conteúdo tecnológico ou grande valor, o conhecimento das técnicas de previsão mais adequadas pode diminuir a dificuldade dos trabalhos de previsão, melhorando a qualidade das decisões intrínsecas ao desenvolvimento de novos produtos.

\section{REFERÊNCIAS BIBLIOGRÁFICAS}

ARMSTRONG, J. S. Research on forecasting: a quarter-century review, 1960-1984. Interfaces. Linthicum, v. 16, n. 1, p. 89-103, 1986.

ARMSTRONG, J. S. Evaluating forecasting methods. In: ARMSTRONG, J. S. Principles of Forecasting: Handbook for Researchers and Practitioners. Norwell: Kluwer Academic Publishers, 2001. p. 365-82.

ARMSTRONG, J. S.; BRODIE, R. Forecasting for Marketing. In: HOOLEY, G. J.; HUSSEY, M. K. Quantitative Methods in Marketing. London: International Thompson Business Press, 1999. p. 92-119.

BASS, F. M. A new product growth model for consumer durables. Management Science, v. 16, n. 15, p. $215-27,1969$.

DALKEY, N.; HELMER, O. An experimental application of the Delphi method to the use of experts. Management Science, v. 9, n. 3, p. 458-67, 1963.

ETZEL, M. J.; WALKER, B. J.; STANTON, W. J. Marketing. São Paulo: Makron Books, 2001.

GALTON, F. Family likeness in stature. Proceedings of Royal Society. London, v. 40, p. 42-72, 1886.

JAIN, C. L. Which forecasting model should we use? Journal of Business Forecasting Methods \& Systems, v. 16, n. 3, p. 2-5, 2000.

KAHN, K. B. An exploratory investigation of new product forecasting practices. The Journal of Product Innovation Management, v. 19, n. 2, p. 133-43, 2002.

KAHN, K. B.; MENTZER, J. T. Forecasting in consumer and industrial markets. The Journal of Business Forecasting, v. 14, n. 2, p. 21-8, 1995.

KOTLER, P. Administração de Marketing. São Paulo: Prentice Ha11, 2000.

LYNN, G. S.; SCHNAARS, S. P.; SKOV, R. B. Survey of new product forecasting practices in industrial high technology and low technology businesses. Industrial Marketing Management, v. 28, n. 6. p. 565-71, 1999.

MAHAJAN, V.; MULLER, E.; BASS, F. M. New product diffusion models in marketing: a review and directions for research. Journal of Marketing, v. 54, n. 1, p. 1-26, 1990.

MAKRIDAKIS, S.; WHEELWRIGHT, S. C.; HYNDMAN, R. J. Forecasting methods for management. New York: John Wiley \& Sons, 1998.

MATTAR, F. N.; SANTOS, D. G. Gerência de produtos: como tornar seu produto um sucesso. São Paulo: Atlas, 2003.

MENTZER, J. T.; KAHN, K. B. Forecasting technique familiarity, satisfaction, usage and application. Journal of Forecasting, v. 14, n. 5, p. 465-76, 1995. 


\section{SELEÇÃO DE TÉCNICAS DE PREVISÃO DE MERCADO SEGUNDO AS DIFERENTES CATEGORIAS DE NOVOS PRODUTOS \\ Dario Amigo Sampron}

REINMUTH, J. E. Forecasting the impact of a new product introduction. Academy of Marketing Science Journal, v. 2, n. 2, p. 391-400, 1974.

SANDHUSEN, R. L. Marketing básico. São Paulo: Saraiva, 1998.

\section{Artigo recebido em 05.11.2003. Aprovado em 07.04.2005.}

Dario Amigo Sampron (in memoriam)

Ex-professor da Unitau e Univap. 